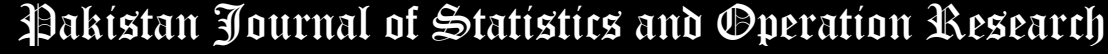

\section{On A New Two Parameter Fréchet Distribution with Applications}

Rania H. M. Abdelkhalek*

* Corresponding Author

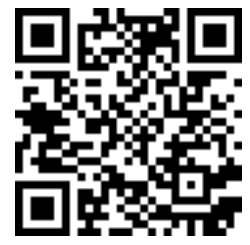

Department of Statistics, Mathematics and Insurance, Benha University, Egypt.

Email: rania.abdelkhalek@fcom.bu.edu.eg

\begin{abstract}
A new flexible extension of the Fréchet model is proposed and studied. Some of its fundamental statistical properties are derived. The importance of the new model is shown via two applications to real data sets. We assess the performance of the maximum likelihood estimations of the new distribution with respect to sample size $\mathrm{n}$. The assessment was based on a simulation study. The new model is much better than other important competitive models.
\end{abstract}

Key Words: Fréchet Distribution; Lomax G family; Modeling Data; Probability Weighted Moments; Order Statistics; Simulation.

Mathematical Subject Classification: 62N02; 62N01; 62E10.

\section{Introduction}

The aim of this paper is to introduce first the generalization of the one parameter Fréchet (Fr) distribution using the one parameter Lomax-G (Lx-G) family originally introduced by Cordeiro et al. (2018). The probability density function (PDF) and cumulative distribution function (CDF) of the one parameter Fr distribution are given by (for $x \geq$ 0)

$$
h_{b}(x)=b x^{-(1+b)} e^{-x^{-b}} \text { and } H_{b}(x)=e^{-x^{-b}}
$$

respectively, where $b>0$ is a shape parameter. Consider the Lx-G family of distributions defined by

$$
F_{\beta, \psi}(x)=1-\left[1+\sigma_{\psi}(x)\right]^{-\beta}
$$

where $\boldsymbol{\sigma}_{\psi}(x)=\frac{H_{\psi}(x)}{\bar{H}_{\psi}(x)}$ and $\psi$ is the parameters vector, $\bar{H}_{\psi}(x)=1-H_{\psi}(x)$ and $H_{\psi}(x)$ is the CDF of the base line distribution. The PDF corresponding to (2) is given by

$$
f_{\beta, \psi}(x)=\beta h_{\psi}(x) \bar{H}_{\psi}(x)^{-2}\left\{1+\left[H_{\psi}(x) / \bar{H}_{\psi}(x)\right]\right\}^{-(1+\beta)},
$$

where $h_{\psi}(x)=d H_{\psi}(x) / d x$ refers to the baseline PDF. The hazard rate function (HRF) of $X$ reduces to $\tau(x)=$ $\beta h_{\psi}(x) \bar{H}_{\psi}(x)^{-2}\left[1+\nabla_{\psi}(x)\right]^{-1}$. Inserting (1) in to (2) we have

$$
F_{\beta, b}(x)=1-\left(1+\frac{e^{-x^{-b}}}{1-e^{-x^{-b}}}\right)^{-\beta}
$$

Equation (4) represents the CDF of the LxFr model. The PDF corresponding to (4) is given by

$$
f_{\beta, b}(x)=\beta b \frac{x^{-(1+b)} e^{-x^{-b}}}{\left(1-e^{-x^{-b}}\right)^{2}}\left(1+\frac{e^{-x^{-b}}}{1-e^{-x^{-b}}}\right)^{-(1+\beta)} .
$$

The LxFr density can be "right-skewed", whereas the LxFr HRF can be "upside" down (see Figure 1). Many new useful Fréchet extensions are introduced by Barreto-Souza et al. (2011), Mahmoud and Mandouh (2013), Korkmaz et 
al. (2017, 2018), Aryal and Yousof (2017), Brito et al. (2017), ul Haq et al. (2017), Chakraborty et al. (2019), Jahanshahi et al. (2019), Yousof et al. (2016, 2017a,b,c, 2018a,b and 2020), Salah et al. (2020) and Al-Babtain et al. (2020a,b). Hereafter, we denote by $X \sim \operatorname{LxFr}(x, \beta, b)$, a RV having density function (5). The CDF (4) of $X$ can be expressed as

$$
F(x)=1-\underbrace{\left(1+\frac{e^{-x^{-b}}}{1-e^{-x^{-b}}}\right)^{-\beta}}_{A(x)}
$$

First, we consider two power series

and

$$
\left.\frac{1}{\left(1+\frac{\xi_{1}}{\xi_{2}}\right)^{\delta}}\right|_{\left(\left|\frac{\xi_{1}}{\xi_{2}}\right|<1, \delta>0\right)}=\sum_{d=0}^{\infty} 2^{-\delta-d}\left(\begin{array}{c}
-\delta \\
d
\end{array}\right)\left(\frac{\xi_{1}}{\xi_{2}}-1\right)^{d}
$$

$$
\left.\frac{1}{\left(1-\frac{\xi_{1}}{\xi_{2}}\right)^{\delta}}\right|_{\left(\left|\frac{\xi_{1}}{\xi_{2}}\right|<1, \delta>0\right)}=\sum_{d=0}^{\infty} \frac{\Gamma(\delta+d)}{d ! \Gamma(\delta)}\left(\frac{\xi_{1}}{\xi_{2}}\right)^{d}
$$

Applying (7) for $A(x)$ in (6) gives

$$
F(x)=1-\sum_{\kappa=0}^{\infty} 2^{-\beta-\kappa}\left(\begin{array}{c}
-\beta \\
\kappa
\end{array}\right)\left(\frac{e^{-x^{-b}}}{1-e^{-x^{-b}}}-1\right)^{\kappa}
$$

Second, using the binomial expansion, the last equation can be expressed as

$$
F(x)=1-\sum_{\kappa=0}^{\infty} \sum_{j=0}^{\kappa}(-1)^{j}\left(\frac{1}{2}\right)^{\beta+\kappa}\left(e^{-x^{-b}}\right)^{(\kappa-j)} \underbrace{\left(1-e^{-x^{-b}}\right)^{-(\kappa-j)}}_{B(x)}\left(\begin{array}{c}
\kappa \\
j
\end{array}\right)\left(\begin{array}{c}
-\beta \\
\kappa
\end{array}\right) .
$$

Third, applying (8) for $B(x)$ in the last equation we get

$$
F(x)=1-\left.\sum_{\zeta, \kappa=0}^{\infty} \sum_{j=0}^{\kappa} a_{j, \zeta, \kappa} \Pi_{\varpi}(x, b)\right|_{\varpi=\kappa+\zeta-j},
$$

where $a_{j, \zeta, \kappa}=\left(\frac{1}{2}\right)^{\beta+\kappa} \frac{(-1)^{j} \Gamma(\varpi)}{\zeta ! \Gamma(\kappa-j)}\left(\begin{array}{c}\kappa \\ j\end{array}\right)\left(\begin{array}{c}-\beta \\ \kappa\end{array}\right)$ and $\Pi_{\varpi}(x, b)=e^{-\varpi x^{-b}}$ is the CDF of the Fr CDF with scale parameter $\varpi^{\frac{1}{b}}$ and shape parameter $b$. By differentiating (9), we obtain

$$
f(x)=\left.\sum_{\zeta, \kappa=0}^{\infty} \sum_{j=0}^{\kappa} C_{j, \zeta, \kappa} \pi_{\varpi}(x, b)\right|_{(\zeta+\kappa \geq 1)},
$$

where $\pi_{\varpi}(x, b)=b \varpi x^{-(1+b)} e^{-\varpi x^{-b}}$ is the Fr density with scale parameter $\varpi^{\frac{1}{b}}$ and shape parameter $b$ and $C_{j, \zeta, \kappa}=$ $-a_{j, \zeta, \kappa}$.

\section{Properties}

\subsection{Moments and generating function}

Let $Z$ be a random variable (RV) having the Fr distribution (1) with parameter $b$. The $\mathrm{r}$ th ordinary and incomplete moments of $Z$ are given by $\mu_{r}^{\prime}=\left.\Gamma\left(1-\frac{r}{b}\right)\right|_{(r<b)}$ and $I_{r}(t)=\left.\gamma\left(1-\frac{r}{b},\left(\frac{1}{t}\right)^{b}\right)\right|_{(r<b)}$ respectively, where $\Gamma(1+\vartheta)=$ $\int_{0}^{\infty} t^{\vartheta} e^{-t} d t, \quad \Gamma(1+\vartheta, \xi)=\int_{\xi}^{\infty} t^{\vartheta} e^{-t} d t \quad$ and $\left.\quad \gamma(\vartheta, \xi)\right|_{(\vartheta \neq 0,-1,-2, \ldots)}=\int_{0}^{\xi} t^{\vartheta-1} e^{-t} d t=\frac{\xi^{\vartheta}}{\vartheta}\left\{1 F_{1}[\vartheta ; \vartheta+1 ;-\xi]\right\}=$ $\sum_{\kappa=0}^{\infty} \xi^{\vartheta+\kappa} \frac{(-1)^{\kappa}}{\kappa !(\vartheta+\kappa)}$ wher $1 F_{1}[\bullet, \bullet, \bullet] \quad$ is a confluent hypergeometric function and $\Gamma(1+\vartheta)=\Gamma(1+\vartheta, \xi)+$ $\gamma(1+\vartheta, \xi)$. The $\mathrm{r}$ th ordinary moment of $X$ say $\mu_{r}^{\prime}=E\left(X^{r}\right)$, is determined from (10) as

$$
\mu_{r}^{\prime}=\left.\Gamma\left(1-\frac{r}{b}\right) \sum_{\zeta, \kappa=0}^{\infty} \sum_{j=0}^{\kappa} C_{j, \zeta, \kappa} \varpi^{\frac{r}{b}}\right|_{(\zeta+\kappa \geq 1 \text { and } r<b)} .
$$

The rth incomplete moment of $X$, say $I_{r}(t)$, can be determined from (10) as 


$$
I_{r}(t)=\int_{-\infty}^{t} x^{r} f(x) d x=\left.\gamma\left(1-\frac{r}{b},\left(\frac{1}{t}\right)^{b}\right) \sum_{\substack{\zeta, \kappa=0 \\ \mid(\zeta+\kappa \geq 1)}}^{\infty} \sum_{j=0}^{\kappa} C_{j, \zeta, \kappa} \varpi^{\frac{r}{b}}\right|_{(r<b)} .
$$

The moment generating function (mgf) $M(t)=E\left(e^{t X}\right)$ of $X$ follows from (10) as

$$
M(t)=\left.\Gamma\left(1-\frac{r}{b}\right) \sum_{\zeta, \kappa, r=0}^{\infty} \sum_{j=0}^{\kappa} C_{j, \zeta, \kappa} \frac{t^{r}}{r !} \varpi^{\frac{r}{b}}\right|_{(\zeta+\kappa \geq 1 \text { and } r<b)} .
$$

\subsection{Probability weighted moments (PWMs)}

The (s,r) th PWM of $X$ denoted by $\rho_{s, r}$ is formally defined by $\rho_{s, r}=E\left\{X^{s} F(X)^{r}\right\}=\int_{-\infty}^{\infty} x^{s} F(x)^{r} f(x) d x$.

Consider the Taylor series $z^{\beta}=\sum_{d=0}^{\infty} \beta_{[d]} \frac{1}{d !}(z-1)^{d}=\sum_{j=0}^{\infty} z^{j} \tau_{j}(\beta)$ where $\beta_{[d]}=\beta(\beta-1) \ldots(\beta-d+1)$ is the descending factorial and $\tau_{j}(\beta)=\sum_{d=j}^{\infty}\left(\begin{array}{c}d \\ j\end{array}\right) \frac{(-1)^{d-j}}{d !} \beta_{[d]}$. First, applying the Taylor series in $z^{\beta}$ for $F(x)^{r}$, we obtain

$$
F(x)^{r}=\sum_{j=0}^{\infty}(-1)^{j} \tau_{j}(r)\left(1+\frac{e^{-x^{-b}}}{1-e^{-x^{-b}}}\right)^{-j \beta} .
$$

Second, using (5) and the last equation, we have

$$
f(x) F(x)^{r}=\beta b x^{-(1+b)} e^{-x^{-b}}\left\{1-e^{-x^{-b}}\right\}^{-2} \sum_{j=0}^{\infty}(-1)^{j} \tau_{j}(r) \underbrace{\left(1+\frac{e^{-x^{-b}}}{1-e^{-x^{-b}}}\right)^{-(j+1) \beta-1}} .
$$

Applying (7) for $C(x)$ in the last equation, we obtain

$$
f(x) F(x)^{r}=\sum_{j, \kappa=0}^{\infty} \frac{(-1)^{j} \tau_{j}(r) \beta b e^{-x^{-b}}}{2^{(j+1) \beta+\kappa+1} x^{b+1}\left(1-e^{-x^{-b}}\right)^{2}} \underbrace{\left(\frac{e^{-x^{-b}}}{1-e^{-x^{-b}}}-1\right)^{\kappa}}_{D(x)}\left(\begin{array}{c}
-(j+1) \beta-1 \\
\kappa
\end{array}\right) .
$$

Third, using the binomial expansion for $D(x)$, the last equation can be rewritten as

$$
f(x) F(x)^{r}=\beta b \sum_{j, \kappa=0}^{\infty} \sum_{\zeta=0}^{\kappa}(-1)^{j+\zeta} \frac{\tau_{j}(r)\left(e^{-x^{-b}}\right)^{1+\kappa-\zeta}}{2^{(j+1) \beta+\kappa+1} x^{1+b}}\left(\begin{array}{c}
\kappa \\
\zeta
\end{array}\right)\left(\begin{array}{c}
-(j+1) \beta-1 \\
\kappa
\end{array}\right) \underbrace{\left(1-e^{-x^{-b}}\right)^{-[\kappa-\zeta+2]}}_{E(x)} .
$$

Applying (8) for $E(x)$ in the last equation gives

$$
f(x) F(x)^{r}=\left.\sum_{\kappa, m=0}^{\infty} \sum_{\zeta=0}^{\kappa} c_{\zeta, \kappa, m}^{(r)} \pi_{\vartheta}(x ; b)\right|_{\vartheta=1+\kappa-\zeta+m}
$$

where $c_{\zeta, \kappa, m}^{(r)}=\beta V_{\zeta, \kappa, m} f_{j}(r), \tau_{j}(r)$ as defined in (11) and

$$
V_{\zeta, \kappa, m}=\left.\sum_{j=0}^{\infty}\left(\begin{array}{c}
\kappa \\
\zeta
\end{array}\right)\left(\begin{array}{c}
-(j+1) \beta-1 \\
\kappa
\end{array}\right) \frac{(-1)^{j+\zeta}(\kappa-\zeta+2)^{(m)}}{2^{(j+1) \beta+\kappa+1} \vartheta m !}\right|_{(\zeta \leq \kappa)}
$$

where $q^{(n)}=\Gamma(q+n) / \Gamma(q)$ denotes the rising factorial. Finally, the (s,r) th PWM of $X$ can be determined as

$$
\rho_{s, r}=\left.\Gamma\left(1-\frac{s}{b}\right) \sum_{\kappa, m=0}^{\infty} \sum_{\zeta=0}^{\kappa} c_{\zeta, \kappa, m}^{(r)} \vartheta^{\frac{s}{b}}\right|_{(\zeta \leq \kappa \text { and } s<b)} .
$$

\subsection{Residual life and reversed residual life functions}

The $\mathrm{n} t h$ moment of the residual life, say $w_{n}(t)=\left.E\left[(X-t)^{n}\right]\right|_{(X>t, n=1,2, \ldots)}$ uniquely determines $F(x)$. The $\mathrm{n} t h$ moment of the residual life of $X$ is given by

Therefore

$$
w_{n}(t)=\frac{\int_{t}^{\infty}(x-t)^{n} d F(x)}{1-F(t)} .
$$




$$
w_{n}(t)=\left.\frac{\Gamma\left(1-\frac{n}{b},\left(\frac{1}{t}\right)^{b}\right)}{1-F(t)} \sum_{\zeta, \kappa=0}^{\infty} \sum_{j=0}^{\kappa} C_{j, \zeta, \kappa}^{(m)} \varpi^{n}\right|_{(\zeta+\kappa \geq 1 \text { and } n<b)},
$$

where $C_{j, \zeta, \kappa}^{(m)}=C_{j, \zeta, \kappa}(1-t)^{n}$. The mean residual life (MRL) function or the life expectation at age $t$ is defined by $\left.w_{1}(t)\right|_{(X>t, n=1)}=E[(X-t)]$ which represents the expected additional life length for a unit which is alive at age $t$. The MRL of $X$ can be obtained by setting $n=1$ in the last equation. The $\mathrm{n} t h$ moment of the reversed residual life, say $W_{n}(t)=\left.E\left[(t-X)^{n}\right]\right|_{(t>0, X \leq t}$ and $\left.n=1,2, \ldots\right)$,

uniquely determines $F(x)$. We obtain

$$
W_{n}(t)=\frac{\int_{0}^{t}(t-x)^{n} d F(x)}{F(t)}
$$

Then, the $\mathrm{n}$ th moment of the reversed residual life of $X$ is

$$
W_{n}(t)=\left.\frac{\gamma\left(1-\frac{n}{b},\left(\frac{1}{t}\right)^{b}\right)}{F(t)} \sum_{\zeta, \kappa=0}^{\infty} \sum_{j=0}^{\kappa} C_{j, \zeta, \kappa}^{(M)} \varpi^{\frac{n}{b}}\right|_{(\zeta+\kappa \geq 1 \text { and } n<b)}
$$

where $C_{j, \zeta, \kappa}^{(M)}=C_{j, \zeta, \kappa} \sum_{r=0}^{n}(-1)^{r}\left(\begin{array}{l}n \\ r\end{array}\right) t^{n-r}$. The mean inactivity time (MIT), also called the mean reversed residual life function, is given by $W_{1}(t)=\left.E[(t-X)]\right|_{(t>0, X \leq t}$ and $\left.n=1\right)$,

and it represents the waiting time elapsed since the failure of an item on condition that this failure had occurred in $(0, t)$.

\subsection{Order statistics}

Let $X_{1}, \cdots, X_{n}$ be a random sample (RS) from the LxFr and let $X_{1}:{ }_{n}, \cdots, X_{n}: n$ be the corresponding order statistics. The PDF of the ith order statistic, say $X_{i}: n$, is given by

$$
f_{i}: n(x)=\frac{f(x)}{B(i, 1+n-i)} \sum_{r=0}^{n-i}(-1)^{r}\left(\begin{array}{c}
n-i \\
r
\end{array}\right) F^{r+i-1}(x),
$$

where $B(\bullet, \bullet)$ is the beta function, then we can write

$$
f(x) F(x)^{r+i-1}=\sum_{\kappa, m=0}^{\infty} \sum_{\zeta=0}^{\kappa} C_{\zeta, \kappa, m}^{(r+i-1)} \pi_{\vartheta}(x ; b),
$$

where $C_{\zeta, \kappa, m}^{(r+i-1)}$ is as defined before. So, the PDF of $X_{i}: n$ becomes using the last expression as

$$
f_{i}: n(x)=\frac{1}{B(i, 1+n-i)} \sum_{\kappa, m=0}^{\infty} \sum_{r=0}^{n-i} \sum_{\zeta=0}^{\kappa} c_{\zeta, \kappa, m}^{(r+i-1)}(-1)^{r}\left(\begin{array}{c}
n-i \\
r
\end{array}\right) \pi_{\vartheta}(x ; b) .
$$

Then, the density function of the LxFr order statistics is a linear combination of the Fr density. Based on this equation, the properties of $X_{i}: n$ can be easily determined from those properties of the Fr density. Then The q $t h$ ordinary moment of $X_{i}: n$ say $E\left(X_{i}^{\xi}: n\right)$, is determined from (12) as

$$
E\left(X_{i: n}^{\xi}\right)=\left.\Gamma\left(1-\frac{\xi}{b}\right) \sum_{\kappa, m=0}^{\infty} \sum_{r=0}^{n-i} \sum_{\zeta=0}^{\kappa} \frac{(-1)^{r}\left(\begin{array}{c}
n-i \\
r
\end{array}\right) C_{\zeta, \kappa, m}^{(r+i-1)}}{B(i, 1+n-i) \vartheta^{\frac{-\xi}{b}}}\right|_{(\xi<b)} .
$$

3.Numerical analysis for the $E(X), \operatorname{Var}(X), \operatorname{Ske}(X)$ and $\operatorname{Ku}(X)$ measures

Numerical analysis for the $E(X), \operatorname{Var}(X), \operatorname{Ske}(X)$ and $\operatorname{Ku}(X)$ are calculated in Table 2 and 3 using the well-known relationships for some selected values of parameters using the R software. Based on Tables 2 and 3 we note that, the skewness of the LxFr distribution can range in the interval $(\mathbf{- 2 7 2 . 6 8}, \mathbf{6 1 . 4 3})$, whereas the skewness of the Fr distribution varies only in the interval (1.2, 3.5). Further, the spread for the LxFr kurtosis is ranging from 3.932 to 5058.65, whereas the spread for the Fr kurtosis only varies from 5.7 to 48.1 with the above parameter values.

4.Maximum likelihood estimation

Let $x_{1}, \ldots, x_{n}$ be a RS from the LxFr model with parameters $\beta$ and $b$. For determining the MLE, we have the following log-likelihood function 


$$
\ell=\ell(\underline{\Theta})=n \log \beta+n \log b-(b+1) \sum_{i=1}^{n} x_{i}-\sum_{i=1}^{n} x^{-b}-2 \sum_{i=1}^{n} \log \left(1-s_{i}\right)-(\beta+1) \sum_{i=1}^{n} \log z_{i},
$$

where $s_{i}=e^{-x^{-b}}$ and $z_{i}=\left[1+\left(\frac{s_{i}}{1-s_{i}}\right)\right]$. The score vector is given as

$$
I_{(\beta)}=\frac{n}{\beta}-\sum_{i=1}^{n} \log z_{i}, I_{(b)}=\frac{n}{b}+n \log -\sum_{i=1}^{n} x_{i}-\sum_{i=1}^{n} w_{i}-2 \sum_{i=1}^{n} \frac{w_{i} s_{i}}{1-s_{i}}-(\beta+1) \sum_{i=1}^{n} \frac{t_{i}}{z_{i}},
$$

where $m_{i}=-b \frac{s_{i}}{x_{i}}\left(x^{-1}\right)^{b-1}\left[1-s_{i}\right]^{-2}, t_{i}=-\frac{w_{i} s_{i}}{\left[1-s_{i}\right]^{2}}$ and $w_{i}=\left(x^{-1}\right)^{b} \log \left(x^{-1}\right)$. Setting the nonlinear system of equations $I_{(\beta)}=0$ and $I_{(b)}=0$ and solving them simultaneously yields the MLE. To solve these equations, it is usually more convenient to use nonlinear optimization methods such as the quasi-Newton algorithm to numerically maximize $\ell$.

\section{Simulation studies}

We simulate the LxFr model via taking $n=20,50,150,500$ and 1000 . Then, repeating this process $N=1000$ times and calculate the averages of the estimates (AEs), mean squared errors (MSEs). Table 2 gives all simulation results. The numerical results in Table 1 indicate that both MSEs and Biases decay toward 0 when $n$ increases for all initial values of of $\beta$ and $b$. The AEs of the parameters tend to be closer to the true (initial) parameter values (I: $\beta=0.75$ and $b=2.25$ and II: $\beta=1.5$ and $b=1.25$ ) when $n$ increases. These results support that the asymptotic normal model provides an adequate approximation to the finite sample distribution of the MLEs. Table 1 below gives the AEs, MSEs and Bias based on $N=1000$ simulations of the LxFr model for some values of $\beta$ and $b$.

\section{Real data modeling}

This section presents two applications of the new distribution using real data sets. We shall compare the fit of the new distribution with the Weibull Inverse Weibull (W-Fr), exponentiated Fr (E-Fr), Kumaraswamy Fr (Kum-Fr), beta Fr (B-Fr) transmuted Fr (T-Fr), gamma extended Fr (GE-Fr), Marshall-Olkin Fr (MO-Fr), MOKum-Fr, generalized MOFr(GMO-Fr), KumMO-Fr and Fr distributions. The PDFs of the competitive model are available in statistical literature.

The $1^{\text {st }}$ data set consists of 100 observations of breaking stress of carbon fibres (in Gba) given by Nichols and Padgett (2006): (0.92, 1.183, 1.187, 1.192, 1.196, 1.213, 1.215, 1.2199, 1.22, 1.224, 1.225, 1.228, 1.237, 1.24, 1.628, 1.684, $1.711,1.718,0.928,0.997,0.9971,1.061,1.117,1.162,1.733,1.738,1.892,1.944,2.035,2.037,2.043,2.046,2.059$, $2.111,1.471,1.475,1.477,1.48,1.489,1.244,1.259,1.261,1.263,1.276,1.5304,1.533,1.544,1.5443,1.552,1.556$, $1.562,1.566,1.585,1.586,1.599,1.602,1.614,1.616,1.617,1.449,1.4497,1.45,1.459,1.515,1.53,1.501$, $1.507,1.743,1.759,1.777,1.794,1.31,1.321,1.329,1.331,1.337,1.351,1.359,1.388,1.408,1.799,1.806,1.814$, $1.816,1.828,1.83,1.884,2.165,2.686,2.778,2.972,3.504,1.972,1.984,1.987,2.02,2.0304,2.029,3.863,5.306)$.

The $2^{\text {nd }}$ data set consists of 63 observations of the strengths of $1.5 \mathrm{~cm}$ glass fibres given by Smith and Naylor (1987): (1.014, 1.248, 1.267, 1.271, 1.272, 1.275, 1.276, 1.355, 1.361, 1.364, 1.379, 1.409, 1.426, 1.459, 2.456, 2.592, $1.292,1.081,1.082,1.185,1.223,1.304,1.306,1.46,1.476,1.481,1.484,1.501,1.506,1.524,1.526,1.535,1.541$, $1.568,1.735,1.278,1.286,1.288,1.867,1.876,1.878,1.91,1.916,1.972,2.012,1.747,1.748,1.757,1.800,1.579$, $1.581,1.591,1.593,1.602,1.666,1.67,1.684,1.691,1.704,1.731,1.806,3.197,4.121)$, originally obtained by workers at the UK National Physical Laboratory. Many other new real data sets are available in Yousof et al. (2019), Elgohari Yousof (2020) Ali et al. (2021a, b) and Ibrahim et al. (2020). We consider the following criteria: the maximized Log-Likelihood, AIC (Akaike Information Criterion), CAIC (Consistent Akaike Information Criterion), BIC (Bayesian Information Criterion) and HQIC (Hannan-Quinn Information Criterion). The model with minimum values for these statistics could be chosen as the best model to fit the data. All results are obtained using the $\mathbf{R}$ PROGRAM. Table 5 and 7 give the MLEs and their standard errors (in parentheses) for the $1^{\text {st }}$ and $2^{\text {nd }}$ data respectively. Tables 4 and 6 compare the LxFr model with other important competitive distributions. The LxFr model gives the lowest values for the AIC, BIC, HQIC and CAIC statistics (in bold values) among all fitted models to these data. So, it may be considered as the best model among them. Figures 2 (first row) gives the TTT plots. Figures 2 (second row) gives the box plots. Figures 2 (third row) gives the normal quantile-quantile (Q-Q) plots. Based on Figures 2 (first row), we note that the HRF for the two data sets are "increasing". Figures 2 (second row), it is noted that the two data sets have some outlier values. The same results are obtained using Figures 2 (third row). Figures 3 7, respectively, display the P-P plots, estimated PDFs, Estimated CDFs, Kaplan-Meier survival Plots and estimated 
HRFs for the proposed model for the $1^{\text {st }}$ and $2^{\text {nd }}$ data. These plots reveal that the proposed distribution yields a better fit than other nested and non-nested models for both data sets.

\section{Conclusions}

A new flexible extension of the IR model is proposed and studied. Some of its fundamental statistical properties are derived such as quantile, moments, incomplete moments and moment generating function. We assessed the performance of the maximum likelihood estimators via a simulation study. The importance of the new model is shown via two applications to real data sets. The new model is much better than other important competitive models (the Weibull Inverse Weibull, exponentiated Inverse Weibull, Kumaraswamy Inverse Weibull, beta Inverse Weibull, transmuted Inverse Weibull, gamma extended Inverse Weibull, Marshall-Olkin Inverse Weibull, Marshall-Olkin Kumaraswamy Inverse Weibull, generalized Marshall-Olkin Inverse Weibull, Kumaraswamy Marshall-Olkin Inverse Weibull and Inverse Weibull distributions) based on two real data sets. As a future work, we can apply many new useful goodness-of-fit tests for right censored validation such as the Nikulin-Rao-Robson goodness-of-fit statistical test, modified Nikulin-Rao-Robson goodness-of-fit statistical test, Bagdonavicius-Nikulin goodness-of-fit statistical test and also modified Bagdonavicius-Nikulin goodness-of-fit statistical test to the new BuXENH model as performed by Ibrahim et al. (2019), Goual et al. (2019, 2020), Mansour et al. (2020a,b,c,d), Yadav et al. (2020) and Goual and Yousof (2020), among others. However, many types of copulas can be used and applied for deriving many new bivariate models based on the new distribution (see Elgohari and Yousof (2020), Mansour et al. (2020e, f) and ElMorshedy et al. (2021) for more details).

\section{References}

1. Al-Babtain, A. A. Elbatal, I. and Yousof, H. M. (2020a). A new three parameter Fréchet model with mathematical properties and applications. Journal of Taibah University for Science, 14(1), 265-278.

2. Al-Babtain, A. A., Elbatal, I. and Yousof, H. M. (2020b). A New Flexible Three-Parameter Model: Properties, Clayton Copula, and Modeling Real Data. Symmetry, 12(3), 440.

3. Ali, M. M., Yousof, H. M. and Ibrahim, M. (2021a). A new version of the generalized Rayleigh distribution with copula, properties, applications and different methods of estimation. Optimal Decision Making in Operations Research \& Statistics: Methodologies and Applications, CRC Press, To appear.

4. Ali, M. M., Ibrahim, M. and Yousof, H. M. (2021a). Expanding the Burr X model: properties, copula, real data modeling and different methods of estimation. Optimal Decision Making in Operations Research \& Statistics: Methodologies and Applications, CRC Press, To appear.

5. Aryal, G. R. and Yousof, H. M. (2017). The exponentiated generalized-G Poisson family of distributions. Economic Quality Control, 32, 1-17.

6. Barreto-Souza, W. M., Cordeiro, G. M. and Simas, A. B. (2011). Some results for beta Fréchet distribution. Communication in Statistics-Theory and Methods, 40, 798-811.

7. Brito, E., Cordeiro, G. M., Yousof, H. M., Alizadeh, M. and Silva, G. O. (2017). Topp-Leone Odd LogLogistic Family of Distributions, Journal of Statistical Computation and Simulation, 87(15), 3040-3058.

8. Chakraborty, S., Handique, L., Altun, E. and Yousof, H. M. (2019). A New Statistical Model for Extreme Values: Properties and Applications. Int. J. Open Problems Compt. Math, 12(1).

9. Cordeiro, G. M., Yousof, H. M., Ramires, T. G. and Ortega, E. M. M. (2018). The Burr XII system of densities: properties, regression model and applications. Journal of Statistical Computation and Simulation, $88(3), 432-456$.

10. Elgohari, H. and Yousof, H. M. (2020). New Extension of Weibull Distribution: Copula, Mathematical Properties and Data Modeling. Statistics, Optimization \& Information Computing, 8(4), 972-993. https://doi.org/10.19139/soic-2310-5070-1036

11. El-Morshedy, M., Alshammari, F. S., Hamed, Y. S., Eliwa, M. S., Yousof, H. M. (2021). A New Family of Continuous Probability Distributions. Entropy, 23, 194. https://doi.org/10.3390/e23020194

12. Goual, H., Yousof, H. M., \& Ali, M. M. (2019). Validation of the odd Lindley exponentiated exponential by a modified goodness of fit test with applications to censored and complete data. Pakistan Journal of Statistics and Operation Research, 15(3), 745-771.

13. Goual, H., \& Yousof, H. M. (2020). Validation of Burr XII inverse Rayleigh model via a modified chisquared goodness-of-fit test. Journal of Applied Statistics, 47(3), 393-423.

14. Goual, H., Yousof, H. M., \& Ali, M. M. (2020). Lomax inverse Weibull model: properties, applications, and a modified Chi-squared goodness-of-fit test for validation. Journal of Nonlinear Sciences \& Applications (JNSA), 13(6), 330-353. 
15. Ibrahim, M., Altun, E. and Yousof, H. M. (2020). A new distribution for modeling lifetime data with different methods of estimation and censored regression modeling. Statistics, Optimization \& Information Computing, 8(2), 610-630.

16. Ibrahim, M., Yadav, A. S., Yousof, H. M., Goual, H. and Hamedani, G. G. (2019). A new extension of Lindley distribution: modified validation test, characterizations and different methods of estimation. Communications for Statistical Applications and Methods, 26(5), 473-495.

17. Jahanshahi, S.M.A., Yousof, H. M. and Sharma, V.K. (2019). The Burr X Fréchet Model for Extreme Values: Mathematical Properties, Classical Inference and Bayesian Analysis. Pak. J. Stat. Oper. Res., 15(3), 797818 .

18. Korkmaz, M. C., Yousof, H. M. and Ali, M. M. (2017). Some theoretical and computational aspects of the odd Lindley Fréchet distribution. İstatistikçiler Dergisi: İstatistik ve Aktüerya, 10(2), 129-140.

19. Korkmaz, M. C., Yousof, H. M., Hamedani G. G. and Ali, M. M. (2018). The Marshall--Olkin generalized G Poisson family of distributions, Pakistan Journal of Statistics, 34, 251-267.

20. Mahmoud, M. R. and Mandouh, R. M. (2013). On the transmuted Fréchet distribution. Journal of Applied Sciences Research, 9, 5553-5561.

21. Mansour, M. M., Ibrahim, M., Aidi, K., Shafique Butt, N., Ali, M. M., Yousof, H. M. and Hamed, M. S. (2020a). A New Log-Logistic Lifetime Model with Mathematical Properties, Copula, Modified Goodnessof-Fit Test for Validation and Real Data Modeling. Mathematics, 8(9), 1508.

22. Mansour, M. M., Butt, N. S., Ansari, S. I., Yousof, H. M., Ali, M. M. and Ibrahim, M. (2020b). A new exponentiated Weibull distribution's extension: copula, mathematical properties and applications. Contributions to Mathematics, 1 (2020) 57-66. DOI: 10.47443/cm.2020.0018

23. Mansour, M., Korkmaz, M. Ç., Ali, M. M., Yousof, H. M., Ansari, S. I. and Ibrahim, M. (2020c). A generalization of the

24. exponentiated Weibull model with properties, Copula and application. Eurasian Bulletin of Mathematics, 3(2), 84-102.

25. Mansour, M., Rasekhi, M., Ibrahim, M., Aidi, K., Yousof, H. M. and Elrazik, E. A. (2020d). A New Parametric Life Distribution with Modified Bagdonavičius-Nikulin Goodness-of-Fit Test for Censored Validation, Properties, Applications, and Different Estimation Methods. Entropy, 22(5), 592.

26. Mansour, M., Yousof, H. M., Shehata, W. A. and Ibrahim, M. (2020e). A new two parameter Burr XII distribution: properties, copula, different estimation methods and modeling acute bone cancer data. Journal of Nonlinear Science and Applications, 13 (5), 223-238.

27. Mansour, M. M., Butt, N. S., Yousof, H. M., Ansari, S. I. and Ibrahim, M. (2020f). A Generalization of Reciprocal Exponential Model: Clayton Copula, Statistical Properties and Modeling Skewed and Symmetric Real Data Sets. Pakistan Journal of Statistics and Operation Research, 16(2), 373-386.

28. Nichols, M. D, Padgett, W. J. (2006). A Bootstrap control chart for Weibull percentiles. Quality and Reliability Engineering International, 22, 141-151.

29. Salah, M. M., El-Morshedy, M., Eliwa, M. S. and Yousof, H. M. (2020). Expanded Fréchet Model: Mathematical Properties, Copula, Different Estimation Methods, Applications and Validation Testing. Mathematics, 8(11), 1949.

30. Smith, R. L. and Naylor, J. C. (1987). A comparison of maximum likelihood and Bayesian estimators for the three-parameter Weibull distribution. Applied Statistics, 36, 358-369.

31. ul Haq, M. A., Yousof, H. M. and Hashmi, S. (2017). A New Five-Parameter Fréchet Model for Extreme Values. Pakistan Journal of Statistics and Operation Research, 617-632.

32. Yadav, A. S., Goual, H., Alotaibi, R. M., Ali, M. M., \& Yousof, H. M. (2020). Validation of the Topp-LeoneLomax model via a modified Nikulin-Rao-Robson goodness-of-fit test with different methods of estimation. Symmetry, 12(1), 57.

33. Yousof, H. M., Afify, A. Z., Abd El Hadi, N. E., Hamedani, G. G. and Butt, N. S. (2016). On six-parameter Fréchet distribution: properties and applications. Pakistan Journal of Statistics and Operation Research, 281299.

34. Yousof, H. M., Afify, A. Z., Hamedani, G. G. and Aryal, G. (2017a). The Burr X generator of distributions for lifetime data. Journal of Statistical Theory and Applications, 16, 288--305.

35. Yousof, H. M., Altun, E., Rasekhi, M., Alizadeh, M., Hamedani, G. G. and Ali, M. M. (2019). A new lifetime model with regression models, characterizations and applications. Communications in Statistics-Simulation and Computation, 48(1), 264-286. 
36. Yousof, H. M., Alizadeh, M., Jahanshahiand, S. M. A., Ramires, T. G., Ghosh, I. and Hamedani G. G. (2017b). The transmuted Topp-Leone G family of distributions: theory, characterizations and applications, Journal of Data Science. 15, 723-740.

37. Yousof, H. M., Altun, E. and Hamedani, G. G. (2018a). A new extension of Frechet distribution with regression models, residual analysis and characterizations. Journal of Data Science, 16, 743-770.

38. Yousof, H. M., Hamedani, G. G. and Ibrahim, M. (2020). The two-parameter xgamma Frechet distribution: characterizations, copulas, mathematical properties and different classical estimation methods. Contributions to Mathematics, 2(2020), 32-41.

39. Yousof, H. M., Jahanshahi, S. M., Ramires, T. G Aryal, G. R. and Hamedani G. G. (2017c). A new distribution for extreme values: regression model, characterizations and applications. Journal of Data Science, Journal of Data Science, 16(4), 677-706.

40. Yousof, H. M., Rasekhi, M., Altun, E. and Alizadeh, M. (2018b). The extended odd Frechet family of distributions: properties, applications and regression modeling. International Journal of Applied Mathematics and Statistics, 30(1), 1-30.

\section{Appendix}

Table 1: The AEs and MSEs based on $\mathrm{N}=1000$ simulations.

\begin{tabular}{|c|c|c|c|c|c|c|c|c|}
\hline \multirow[t]{2}{*}{$\mathrm{n}$} & & $\Theta$ & $\mathrm{AE}$ & MSE & \multirow[b]{2}{*}{ II } & \multirow[t]{2}{*}{$\Theta$} & \multirow[t]{2}{*}{$\mathrm{AE}$} & \multirow[t]{2}{*}{ MSE } \\
\hline & I & & & & & & & \\
\hline \multirow[t]{2}{*}{20} & & $\beta$ & 0.767711 & 0.712029 & & $\beta$ & 1.493741 & 1.264799 \\
\hline & & $\mathrm{b}$ & 2.270924 & 0.045512 & & $\mathrm{~b}$ & 1.228515 & 0.031795 \\
\hline \multirow[t]{2}{*}{50} & & $\beta$ & 0.732821 & 0.251313 & & $\beta$ & 1.531327 & 0.448642 \\
\hline & & $\mathrm{b}$ & 2.251502 & 0.016001 & & $\mathrm{~b}$ & 1.247365 & 0.013712 \\
\hline \multirow[t]{2}{*}{150} & & $\beta$ & 0.741990 & 0.086703 & & $\beta$ & 1.507432 & 0.150199 \\
\hline & & $\mathrm{b}$ & 2.250411 & 0.005600 & & $\mathrm{~b}$ & 1.248334 & 0.004799 \\
\hline \multirow[t]{2}{*}{500} & & $\beta$ & 0.749823 & 0.025511 & & $\lambda$ & 1.499133 & 0.048501 \\
\hline & & $\mathrm{b}$ & 2.250639 & 0.001601 & & $\mathrm{~b}$ & 1.248421 & 0.001601 \\
\hline \multirow[t]{2}{*}{1000} & & $\beta$ & 0.750543 & 0.000202 & & $\lambda$ & 1.506995 & 0.001300 \\
\hline & & $\mathrm{b}$ & 2.250322 & 0.000831 & & $\mathrm{~b}$ & 1.250605 & 0.000800 \\
\hline
\end{tabular}

Table 2: $\mathrm{E}(\mathrm{X}), \operatorname{Var}(\mathrm{X}), \operatorname{Ske}(\mathrm{X})$ and $\mathrm{Ku}(\mathrm{X})$ of the $\mathrm{LxFr}$ distribution.

\begin{tabular}{cccccc}
\hline$\beta$ & $\mathrm{b}$ & $\mathrm{E}(\mathrm{X})$ & $\operatorname{Var}(\mathrm{X})$ & $\mathrm{Ske}(\mathrm{X})$ & $\mathrm{Ku}(\mathrm{X})$ \\
\hline 0.1 & 1 & 55.33988 & 23285.220 & 3.724110 & 17.47517 \\
0.5 & & 30.09855 & 9612.0230 & 5.753003 & 41.20552 \\
1 & & 6.331539 & 952.58190 & 16.34861 & 353.1249 \\
2 & & 1.384296 & 9.9752140 & 61.42462 & 9910.895 \\
3 & & 0.863045 & 0.8219024 & 19.07495 & 4303.331 \\
4 & & 0.679596 & 0.2719467 & 5.638141 & 251.6553 \\
5 & & 0.583273 & 0.1414107 & 3.471007 & 44.79156 \\
7.5 & & 0.463939 & 0.0552542 & 2.088686 & 13.06347 \\
10 & & 0.405316 & 0.0321406 & 1.617564 & 8.515312 \\
15 & & 0.344309 & 0.0168258 & 1.197615 & 5.823718 \\
20 & & 0.311258 & 0.0113026 & 0.988696 & 4.859133 \\
25 & & 0.289774 & 0.0085326 & 0.857410 & 4.362587 \\
26 & & 0.286308 & 0.0081389 & 0.836483 & 4.290844 \\
1 & 0.1 & 31.76279 & 14539.140 & 5.058660 & 30.59878 \\
& 0.5 & 27.73040 & 9286.9740 & 5.906109 & 43.18067 \\
& 2 & 1.770454 & 10.103760 & 60.31962 & 9659.170 \\
& 3 & 1.354116 & 0.8423072 & 18.42237 & 4096.210 \\
& 4 & 1.225417 & 0.2708058 & 5.576868 & 252.3548 \\
2 & 4.5 & 1.190151 & 0.1842562 & 4.237641 & 90.43181 \\
6 & 5 & 1.164230 & 0.1337614 & 3.535021 & 47.81271 \\
& 2 & 0.867457 & 0.1105648 & 2.297332 & 17.28198 \\
& 3 & 1.002154 & 0.1010142 & 2.371040 & 18.55080 \\
& & 0.829826 & 0.0110319 & 0.654287 & 3.932961 \\
& & & & &
\end{tabular}




\begin{tabular}{cccccc}
4 & 2 & 0.785059 & 0.0632792 & 1.652700 & 9.325199 \\
1.5 & 2.5 & 1.151524 & 0.3663378 & 6.306288 & 423.7328 \\
13 & 2 & 0.592097 & 0.0130044 & -155.5106 & 2341.584 \\
14 & 2 & 0.584195 & 0.0120511 & -166.6440 & 2575.517 \\
15 & 2 & 0.577116 & 0.0112460 & -177.4740 & 2808.60 \\
20 & 2 & 0.550172 & 0.0085689 & -227.7281 & 3954.326 \\
25 & 2 & 0.531715 & 0.0070534 & -272.6826 & 5058.648 \\
\hline
\end{tabular}

Table 3: $\mathrm{E}(\mathrm{X})$, $\operatorname{Var}(\mathrm{X}), \mathrm{Ske}(\mathrm{X})$ and $\mathrm{Ku}(\mathrm{X})$ of the Fr distribution.

\begin{tabular}{ccccc}
\hline $\mathrm{b}$ & $\mathrm{E}(\mathrm{X})$ & $\operatorname{Var}(\mathrm{X})$ & $\mathrm{Ske}(\mathrm{X})$ & $\mathrm{Ku}(\mathrm{X})$ \\
\hline 5 & 1.164230 & 0.13376140 & 3.535072 & 48.09151 \\
6 & 1.128787 & 0.07995778 & 2.805566 & 24.67812 \\
7 & 1.105767 & 0.05327186 & 2.425097 & 17.53402 \\
10 & 1.068629 & 0.02226241 & 1.910339 & 10.97857 \\
15 & 1.043167 & 0.00885801 & 1.605245 & 8.282494 \\
20 & 1.031453 & 0.00473276 & 1.473884 & 7.333494 \\
30 & 1.020374 & 0.00200272 & 1.353566 & 6.562309 \\
40 & 1.015063 & 0.00110017 & 1.296998 & 6.229997 \\
50 & 1.011947 & 0.00069436 & 1.264099 & 6.045237 \\
75 & 1.007874 & 0.00030302 & 1.221374 & 5.814377 \\
90 & 1.006537 & 0.00020916 & 1.207410 & 5.741088 \\
100 & 1.005872 & 0.00016892 & 1.200478 & 5.705176 \\
\hline
\end{tabular}

Table 4: The statistics AIC, BIC, HQIC and CAIC values for breaking stress data.

\begin{tabular}{ccccc}
\hline \multirow{2}{*}{ Model } & \multicolumn{4}{c}{ Measures } \\
\cline { 2 - 5 } & AIC & BIC & HQIC & CAIC \\
\hline LxFr & $\mathbf{1 5 4 . 0}$ & $\mathbf{1 5 9 . 2}$ & $\mathbf{1 5 6 . 1}$ & $\mathbf{1 5 4 . 1}$ \\
W-Fr & 294.5 & 304.9 & 298.7 & 294.9 \\
E-Fr & 295.7 & 303.5 & 298.9 & 296.0 \\
Kum-Fr & 297.1 & 307.5 & 301.3 & 297.5 \\
B-Fr & 311.1 & 321.6 & 315.4 & 311.6 \\
GE-Fr & 312.0 & 332.4 & 316.2 & 312.4 \\
Fr & 348.3 & 353.5 & 350.4 & 348.4 \\
T-Fr & 350.5 & 358.3 & 353.6 & 350.7 \\
MO-Fr & 351.3 & 359.1 & 354.5 & 351.6
\end{tabular}

Table 5: MLEs and their standard errors (in parentheses) for breaking stress of carbon fiber data.

\begin{tabular}{ccccc}
\hline Model & \multicolumn{4}{c}{ Estimates } \\
\hline $\operatorname{Lx} \operatorname{Fr}(\beta, \mathrm{b})$ & $\mathbf{0 . 1 6 8 1}$ & $\mathbf{1 2 . 8 2 4}$ & \\
& $\mathbf{( 0 . 0 4 2 7})$ & $\mathbf{( 3 . 0 2 6})$ & & \\
$\mathrm{W}-\operatorname{Fr}(\alpha, \beta, \mathrm{a}, \mathrm{b})$ & 2.2231 & 0.355 & 6.9721 & 4.9179 \\
& $(11.41)$ & $(0.411)$ & $(113.8)$ & $(3.756)$ \\
$\operatorname{Kum}-\operatorname{Fr}(\alpha, \beta, \mathrm{a}, \mathrm{b})$ & 2.0556 & 0.4654 & 6.2815 & 224.18 \\
& $(0.071)$ & $(0.007)$ & $(0.063)$ & $(0.164)$ \\
$\mathrm{B}-\operatorname{Fr}(\alpha, \beta, \mathrm{a}, \mathrm{b})$ & 1.6097 & 0.4046 & 22.014 & 29.762 \\
& $(2.498)$ & $(0.108)$ & $(21.43)$ & $(17.479)$ \\
$\operatorname{GE}-\operatorname{Fr}(\alpha, \beta, \mathrm{a}, \mathrm{b})$ & 1.3692 & 0.4776 & 27.645 & 17.4581 \\
& $(2.017)$ & $(0.133)$ & $(14.14)$ & $(14.818)$ \\
$\operatorname{E}-\operatorname{Fr}(\alpha, \beta, \mathrm{a})$ & 69.149 & 0.5019 & 145.33 & \\
& $(57.35)$ & $(0.08)$ & $(122.9)$ & \\
$\operatorname{T}-\operatorname{Fr}(\alpha, \beta, \mathrm{a})$ & 1.9315 & 1.7435 & 0.0819 & \\
& $(0.097)$ & $(0.076)$ & $(0.198)$ & \\
$\operatorname{MO}-\operatorname{Fr}(\alpha, \beta, \mathrm{a})$ & 2.3066 & 1.5796 & 0.5988 &
\end{tabular}




$\begin{array}{llll} & (0.498) & (0.161) & (0.3091) \\ \operatorname{Fr}(\alpha, \beta) & 1.8705 & 1.7766 & \\ & (0.112) & (0.113) & \end{array}$

Table 6: The statistics AIC, BIC, HQIC and CAIC values for glass fiber data.

\begin{tabular}{ccccc}
\hline Model & \multicolumn{4}{c}{ Measures } \\
\cline { 2 - 5 } & AIC & BIC & HQIC & CAIC \\
\hline LxFr & $\mathbf{8 5 . 7 1}$ & $\mathbf{9 0 . 0 0}$ & $\mathbf{8 7 . 4 2}$ & $\mathbf{8 5 . 9 3}$ \\
Fr & 97.72 & 102.4 & 99.4 & 97.96 \\
T-Fr & 100.1 & 106.5 & 102.6 & 100.5 \\
MO-Fr & 101.7 & 108.2 & 104.2 & 102.1 \\
\hline
\end{tabular}

Table 7: MLEs and their standard errors for glass fiber data.

\begin{tabular}{cccc}
\hline Model & & Estimates & \\
\hline $\operatorname{Lx} F r(\beta, b)$ & $\mathbf{0 . 0 8 4 3}$ & $\mathbf{2 5 . 6 5 6}$ & \\
& $\mathbf{( 0 . 0 1 1})$ & $\mathbf{( 0 . 0 0 3})$ & \\
$\operatorname{Fr}(\alpha, \beta)$ & 1.2640 & 2.8884 & \\
& $(0.059)$ & $(0.234)$ & \\
$\operatorname{T}-\operatorname{Fr}(\alpha, \beta, \mathrm{a})$ & 1.3068 & 2.7898 & 0.1298 \\
& $(0.034)$ & $(0.165)$ & $(0.208)$ \\
$\operatorname{MO}-\operatorname{Fr}(\alpha, \beta, \mathrm{a})$ & 1.5441 & 2.3876 & 0.4816 \\
& $(0.226)$ & $(0.253)$ & $(0.252)$ \\
\hline
\end{tabular}


Figure 1: Plots of the LxFr PDF and HRF for selected parameter values. 


Figure 2: TTT plots, box plots and Q-Q plots.


Figure 3: P-P plots.
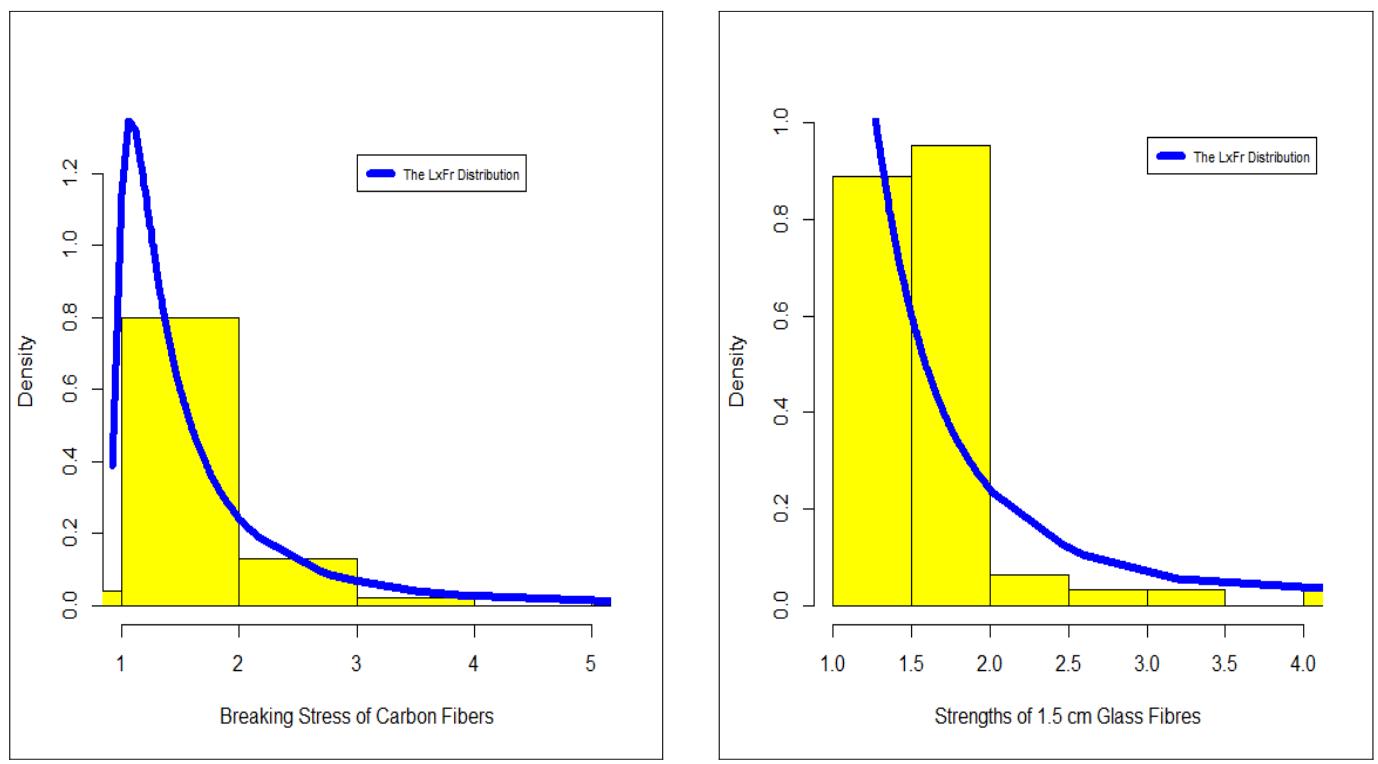

Figure 4: Estimated PDFs. 

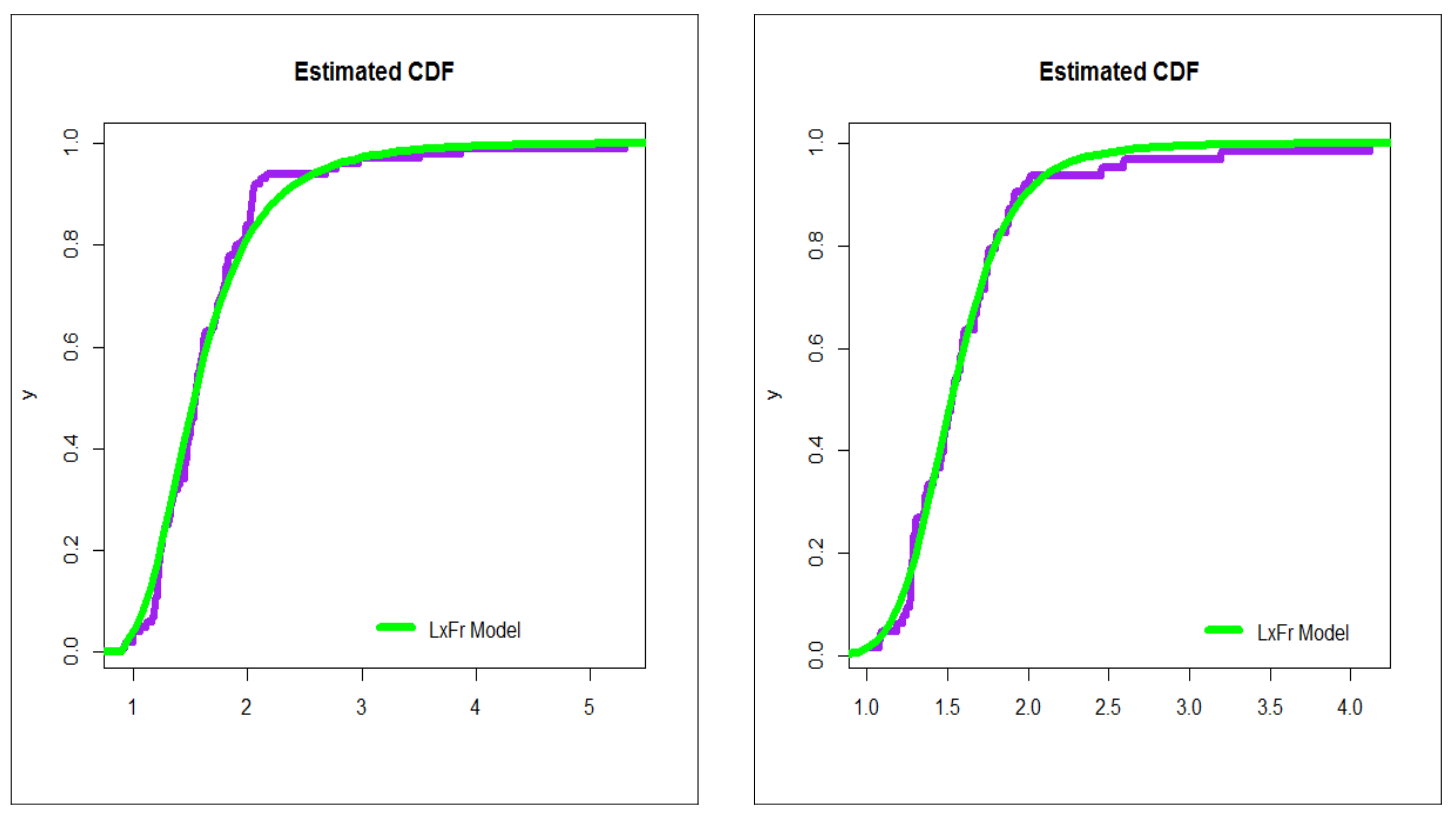

Figure 5: Estimated CDFs.
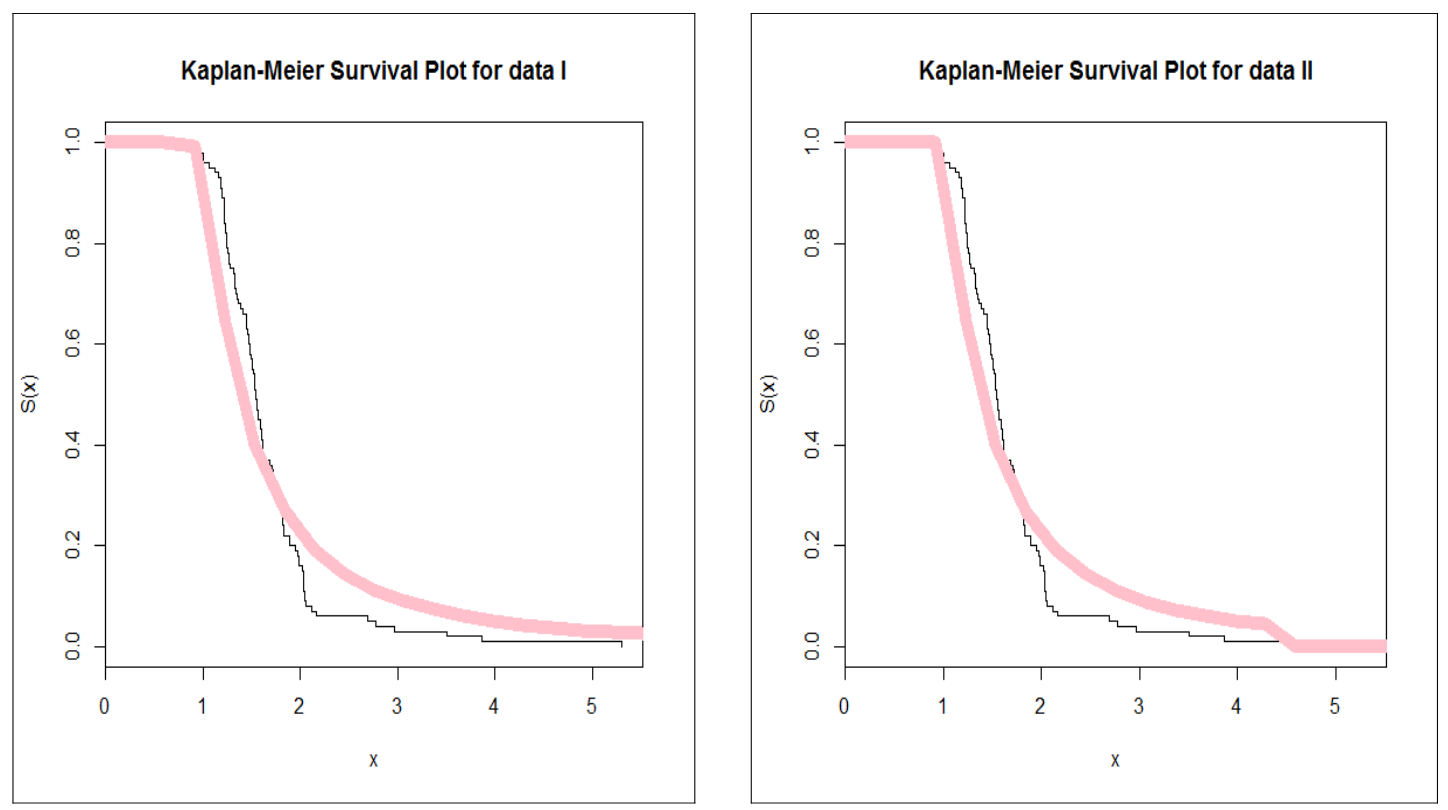

Figure 6: Kaplan-Meier Survival Plots. 



Figure 7: Estimated HRFs. 